\title{
Shift Multiplex Recording of Four-Valued Phase Data Pages by Volume Retardagraphy
}

\author{
Daisuke Barada $^{1,2,3, *}$, Hiroki Sekiguchi ${ }^{2}$, Takashi Fukuda ${ }^{3}$, Shigeo Kawata ${ }^{1,2}$ and \\ Toyohiko Yatagai ${ }^{2}$
}

1 Graduate School of Engineering, Utsunomiya University, 7-1-2 Yoto, Utsunomiya, Tochigi 321-8585, Japan; E-Mail: kwt@cc.utsunomiya-u.ac.jp

2 Center for Optical Research and Education (CORE), Utsunomiya University, 7-1-2 Yoto, Utsunomiya, Tochigi 321-8585, Japan; E-Mails: sh6965.donbon@gmail.com (H.S.); yatagai@cc.utsunomiya-u.ac.jp (T.Y.)

3 Electronics and Photonics Research Institute, National Institute of Advanced Industrial Science and Technology (AIST), Tsukuba Central 5, 1-1-1 Higashi, Tsukuba, Ibaraki 305-5676, Japan; E-Mail: t-fukuda@aist.go.jp

* Author to whom correspondence should be addressed; E-Mail: barada@cc.utsunomiya-u.ac.jp; Tel./Fax: +81-28-689-6094.

Received: 8 December 2013; in revised form: 12 March 2014 / Accepted: 20 March 2014 / Published: 8 April 2014

\begin{abstract}
In this paper, shift multiplex recording of phase data pages on a volume polarization-sensitive medium by retardagraphy is demonstrated. The origin of shift selectivity in volume retardagraphy is explained. In the experiment, four-valued phase data pages are used. Then, a coding method is proposed to correct a reconstructed phase pattern. The recorded phase data pages are reconstructed using the feature of the coding method. By comparing the reconstructed phase data pages with recording phase data pages, symbol error rates of less than $11 \%$ are achieved. From the experimental result, it is verified that volume retardagraphy is applicable to optical memory.
\end{abstract}

Keywords: retardagraphy; polarization holography; volume holographic memory; optical data storage; phase data page; polarization-sensitive material 


\section{Introduction}

Volume holographic memory is expected to optical data storage with huge data capacity and high data transfer rate because many data pages can be recorded on a region in parallel [1]. In holography, a recording beam is split into a signal and a reference beam and two beams are superposed on a recording medium. Therefore, the optical system is complicated and when the length of two optical paths is independently changed by environmental vibration, the quality is reduced. In order to solve the problems, colinear holography has been proposed [2,3]. In colinear holography, signal and reference beams are spatially separated from a recording beam and superposed by using focusing lens. In our previous study, a signal and a reference beam were separated in polarization basis and phase retardation pattern between the signal and the reference beams were recorded on a recording medium. The recording medium is made of a polarization-sensitive material such as azobenzene-containing material [4], photo-crosslinkable polymer [5] and axis-selective photo-reactive molecule doped polymer [6]. That recording technique was named "Retardagraphy" [7-9]. Retardagraphy is based on polarization holography [10-12] so that it is expected that multiplex recording is applicable by retardagraphy. In this paper, shift-multiplex recording is demonstrated by volume retardagraphy.

\section{Principle of Volume Retardagraphy}

In retardagraphy for phase data page recording, the phase of a polarization component is modulated by using a spatial variable retarder such as a parallel-aligned liquid-crystal spatial light modulator (SLM). When the SLM modulates the phase of $p$-polarization component, $p$ - and $s$-polarization components become signal $U_{S}$ and reference $U_{R}$, respectively. The vectorial complex amplitude of a recording beam is represented by

$$
\boldsymbol{U}_{1}(x, y)=\left[\begin{array}{l}
U_{S}(x, y) \\
U_{R}(x, y)
\end{array}\right]
$$

where upper and lower components express $p$ - and $s$-polarization components. The $p$ - and $s$-polarization components can be converted into right- and left-circular polarization components using a quarter-wave plate (QWP) with the fast axis of $45^{\circ}$ as follows:

$$
\begin{gathered}
\boldsymbol{U}_{2}(x, y)=\frac{1}{2}\left[\begin{array}{cc}
1 & -1 \\
1 & 1
\end{array}\right]\left[\begin{array}{cc}
\exp (\mathrm{i} \pi / 4) & 0 \\
0 & \exp (-\mathrm{i} \pi / 4)
\end{array}\right]\left[\begin{array}{cc}
1 & 1 \\
-1 & 1
\end{array}\right]\left[\begin{array}{l}
U_{S}(x, y) \\
U_{R}(x, y)
\end{array}\right] \\
=\frac{U_{\mathrm{S}}(x, y)}{\sqrt{2}}\left[\begin{array}{l}
1 \\
\mathrm{i}
\end{array}\right]+\mathrm{i} \frac{U_{\mathrm{R}}(x, y)}{\sqrt{2}}\left[\begin{array}{c}
1 \\
-\mathrm{i}
\end{array}\right]
\end{gathered}
$$

It is assumed that the vectorial complex amplitude $\boldsymbol{U}_{2}$ is located at an image plane 1 as shown in Figure 1. 
Figure 1. Schematic of optical system of volume retardagraphy. FTL is Fourier transform lens. $(x, y)$ and $\left(x^{\prime}, y^{\prime}\right)$ are coordinates at image planes and a plane between FTL1 and FTL2, respectively.

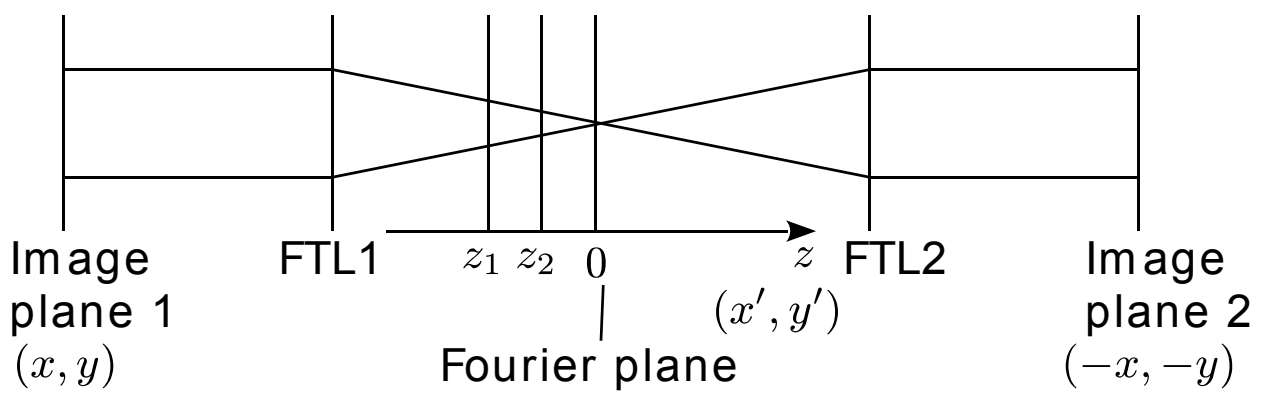

For multiplex recording, selectivity among different data pages is necessary. In this study, shift multiplex recording with a spherical wave is investigated. Therefore, the recording beam must be focused by a lens. Here, the vectorial complex amplitude after the Fourier transform lens (FTL1) is denoted to $\boldsymbol{U}_{3} . \boldsymbol{U}_{3}$ is expressed using arbitrary amplitudes $A_{3, \mathrm{~S}}$ and $A_{3, \mathrm{R}}$, and arbitrary phases $\phi_{3, \mathrm{~S}}$ and $\phi_{3, \mathrm{R}}$ by

$$
\begin{aligned}
& \boldsymbol{U}_{3}\left(x^{\prime}, y^{\prime}\right)=\frac{A_{3, \mathrm{~S}}\left(x^{\prime}, y^{\prime}\right) \exp \left[\mathrm{i} \phi_{3, \mathrm{~S}}\left(x^{\prime}, y^{\prime}\right)\right]}{\sqrt{2}}\left[\begin{array}{l}
1 \\
\mathrm{i}
\end{array}\right]+\frac{A_{3, \mathrm{R}}\left(x^{\prime}, y^{\prime}\right) \exp \left[\mathrm{i} \phi_{3, \mathrm{R}}\left(x^{\prime}, y^{\prime}\right)\right]}{\sqrt{2}}\left[\begin{array}{c}
1 \\
-\mathrm{i}
\end{array}\right] \\
& =\frac{\exp \left[\mathrm{i} \phi_{3}\left(x^{\prime}, y^{\prime}\right)\right]}{2}\left[\begin{array}{cc}
\cos \left[\alpha_{3}\left(x^{\prime}, y^{\prime}\right)\right] & \sin \left[-\alpha_{3}\left(x^{\prime}, y^{\prime}\right)\right] \\
\sin \left[\alpha_{3}\left(x^{\prime}, y^{\prime}\right)\right] & \cos \left[\alpha_{3}\left(x^{\prime}, y^{\prime}\right)\right]
\end{array}\right]\left[\begin{array}{c}
A_{3, \mathrm{~S}}\left(x^{\prime}, y^{\prime}\right)+A_{3, \mathrm{R}}\left(x^{\prime}, y^{\prime}\right) \\
\mathrm{i}\left[A_{3, \mathrm{~S}}\left(x^{\prime}, y^{\prime}\right)-A_{3, \mathrm{R}}\left(x^{\prime}, y^{\prime}\right)\right]
\end{array}\right]
\end{aligned}
$$

where

$$
\begin{gathered}
\phi_{3}\left(x^{\prime}, y^{\prime}\right)=\frac{\phi_{3, \mathrm{~S}}\left(x^{\prime}, y^{\prime}\right)+\phi_{3, \mathrm{R}}\left(x^{\prime}, y^{\prime}\right)}{2} \\
\alpha_{3}\left(x^{\prime}, y^{\prime}\right)=-\frac{\phi_{3, \mathrm{~S}}\left(x^{\prime}, y^{\prime}\right)-\phi_{3, \mathrm{R}}\left(x^{\prime}, y^{\prime}\right)}{2}
\end{gathered}
$$

In general, a polarization-sensitive material exhibits sensitivity for polarization azimuth. When, a polarized beam is illuminated, birefringence is induced in the material. Then, the principal axis of the birefringence and retardance are corresponding to the polarization azimuth and long- and short-axis intensities of elliptical polarization. The polarization azimuth is expressed by Equation 5, and the long- and short-axis intensities $I_{1}$ and $I_{2}$ are expressed by

$$
\begin{aligned}
& I_{1}\left(x^{\prime}, y^{\prime}\right)=\left|A_{3, \mathrm{~S}}\left(x^{\prime}, y^{\prime}\right)+A_{3, \mathrm{R}}\left(x^{\prime}, y^{\prime}\right)\right|^{2} \\
& I_{2}\left(x^{\prime}, y^{\prime}\right)=\left|A_{3, \mathrm{~S}}\left(x^{\prime}, y^{\prime}\right)-A_{3, \mathrm{R}}\left(x^{\prime}, y^{\prime}\right)\right|^{2}
\end{aligned}
$$

Then, two phase holograms corresponding to long- and short-axis of the elliptical polarization are recorded as

$$
\begin{aligned}
& H_{1}\left(x^{\prime}, y^{\prime}\right)=\exp \left\{\mathrm{i}\left[\gamma_{\|} I_{1}\left(x^{\prime}, y^{\prime}\right)+\gamma_{\perp} I_{2}\left(x^{\prime}, y^{\prime}\right)\right]\right\} \\
& H_{2}\left(x^{\prime}, y^{\prime}\right)=\exp \left\{\mathrm{i}\left[\gamma_{\|} I_{2}\left(x^{\prime}, y^{\prime}\right)+\gamma_{\perp} I_{1}\left(x^{\prime}, y^{\prime}\right)\right]\right\}
\end{aligned}
$$

where $\gamma_{\|}$and $\gamma_{\perp}$ are coefficients for the relationship between intensity and phase retardance. Then, the polarization hologram is expressed by 


$$
\begin{aligned}
& H\left(x^{\prime}, y^{\prime}\right) \\
& \quad=\left[\begin{array}{ccc}
\cos \left[\alpha_{3}\left(x^{\prime}, y^{\prime}\right)\right] & -\sin \left[\alpha_{3}\left(x^{\prime}, y^{\prime}\right)\right] \\
\sin \left[\alpha_{3}\left(x^{\prime}, y^{\prime}\right)\right] & \cos \left[\alpha_{3}\left(x^{\prime}, y^{\prime}\right)\right]
\end{array}\right] \\
& \quad \cdot\left[\begin{array}{ccc}
H_{1}\left(x^{\prime}, y^{\prime}\right) & 0 \\
0 & H_{2}\left(x^{\prime}, y^{\prime}\right)
\end{array}\right]\left[\begin{array}{cc}
\cos \left[\alpha_{3}\left(x^{\prime}, y^{\prime}\right)\right] & \sin \left[\alpha_{3}\left(x^{\prime}, y^{\prime}\right)\right] \\
-\sin \left[\alpha_{3}\left(x^{\prime}, y^{\prime}\right)\right] & \cos \left[\alpha_{3}\left(x^{\prime}, y^{\prime}\right)\right]
\end{array}\right]
\end{aligned}
$$

When the polarization hologram is illuminated using a illumination beam $U_{3, \mathrm{R}}{ }^{\prime}$, the vectorial amplitude of the transmitted beam is expressed by

$$
\begin{aligned}
& H\left(x^{\prime}, y^{\prime}\right) U_{3, \mathrm{R}}\left(x^{\prime}, y^{\prime}\right)=\frac{U_{3, \mathrm{R}}\left(x^{\prime}, y^{\prime}\right) \exp \left[-\mathrm{i} \alpha_{3}\left(x^{\prime}, y^{\prime}\right)\right]}{\sqrt{2}}\left[\begin{array}{rr}
\cos \left[\alpha_{3}\left(x^{\prime}, y^{\prime}\right)\right] & -\sin \left[\alpha_{3}\left(x^{\prime}, y^{\prime}\right)\right] \\
\sin \left[\alpha_{3}\left(x^{\prime}, y^{\prime}\right)\right] & \cos \left[\alpha_{3}\left(x^{\prime}, y^{\prime}\right)\right]
\end{array}\right] . \\
& {\left[\begin{array}{c}
\exp \left\{\mathrm{i}\left[\gamma_{\|} I_{1}\left(x^{\prime}, y^{\prime}\right)+\gamma_{\perp} I_{2}\left(x^{\prime}, y^{\prime}\right)\right]\right\} \\
-\operatorname{iexp}\left\{\mathrm{i}\left[\gamma_{\|} I_{2}\left(x^{\prime}, y^{\prime}\right)+\gamma_{\perp} I_{1}\left(x^{\prime}, y^{\prime}\right)\right]\right\}
\end{array}\right]=} \\
& \frac{U_{3, \mathrm{R}^{\prime}}\left(x^{\prime}, y^{\prime}\right) \exp \left\{\mathrm{i}\left[-\alpha_{3}\left(x^{\prime}, y^{\prime}\right)+\left(\gamma_{\|}+\gamma_{\perp}\right) I / 2\right]\right\}}{\sqrt{2}}\left[\begin{array}{cc}
\cos \left[\alpha_{3}\left(x^{\prime}, y^{\prime}\right)\right] & -\sin \left[\alpha_{3}\left(x^{\prime}, y^{\prime}\right)\right] \\
\sin \left[\alpha_{3}\left(x^{\prime}, y^{\prime}\right)\right] & \cos \left[\alpha_{3}\left(x^{\prime}, y^{\prime}\right)\right]
\end{array}\right] \text {. } \\
& {\left[\begin{array}{c}
\exp \left\{\mathrm{i}\left[\left(\gamma_{\|}-\gamma_{\perp}\right) \Delta I\left(x^{\prime}, y^{\prime}\right) / 2\right]\right\} \\
-\operatorname{iexp}\left\{-\mathrm{i}\left[\left(\gamma_{\|}-\gamma_{\perp}\right) \Delta I\left(x^{\prime}, y^{\prime}\right) / 2\right]\right\}
\end{array}\right]=} \\
& \frac{U_{3, \mathrm{R}^{\prime}}\left(x^{\prime}, y^{\prime}\right) \exp \left\{\left[-\alpha_{3}\left(x^{\prime}, y^{\prime}\right)+\left(\gamma_{\|}+\gamma_{\perp}\right) I\left(x^{\prime}, y^{\prime}\right) / 2\right]\right\}}{\sqrt{2}}\left[\begin{array}{cc}
\cos \left[\alpha_{3}\left(x^{\prime}, y^{\prime}\right)\right] & -\sin \left[\alpha_{3}\left(x^{\prime}, y^{\prime}\right)\right] \\
\sin \left[\alpha_{3}\left(x^{\prime}, y^{\prime}\right)\right] & \cos \left[\alpha_{3}\left(x^{\prime}, y^{\prime}\right)\right]
\end{array}\right] \text {. } \\
& \left\{\cos \left[\left(\gamma_{\|}-\gamma_{\perp}\right) \Delta I\left(x^{\prime}, y^{\prime}\right) / 2\right]\left[\begin{array}{c}
1 \\
-\mathrm{i}
\end{array}\right]+\mathrm{i} \sin \left[\left(\gamma_{\|}-\gamma_{\perp}\right) \Delta I\left(x^{\prime}, y^{\prime}\right) / 2\right]\left[\begin{array}{l}
1 \\
\mathrm{i}
\end{array}\right]\right\}= \\
& \frac{U_{3, \mathrm{R}^{\prime}}\left(x^{\prime}, y^{\prime}\right) \exp \left\{\mathrm{i}\left[\left(\gamma_{\|}+\gamma_{\perp}\right) I\left(x^{\prime}, y_{\prime}^{\prime}\right) / 2\right]\right\}}{\sqrt{2}} . \\
& \left\{\cos \left[\frac{\left(\gamma_{\|}-\gamma_{\perp}\right) \Delta I\left(x^{\prime}, y^{\prime}\right)}{2}\right]\left[\begin{array}{c}
1 \\
-\mathrm{i}
\end{array}\right]+\mathrm{i} \exp \left[-\mathrm{i} 2 \alpha_{3}\left(x^{\prime}, y^{\prime}\right)\right] \sin \left[\frac{\left(\gamma_{\|}-\gamma_{\perp}\right) \Delta I\left(x^{\prime}, y^{\prime}\right)}{2}\right]\left[\begin{array}{l}
1 \\
\mathrm{i}
\end{array}\right]\right\}
\end{aligned}
$$

where

$$
\begin{gathered}
I\left(x^{\prime}, y^{\prime}\right)=I_{1}\left(x^{\prime}, y^{\prime}\right)+I_{2}\left(x^{\prime}, y^{\prime}\right) \\
\Delta I\left(x^{\prime}, y^{\prime}\right)=I_{1}\left(x^{\prime}, y^{\prime}\right)-I_{2}\left(x^{\prime}, y^{\prime}\right)
\end{gathered}
$$

When $\left(\gamma_{\|}+\gamma_{\perp}\right) I\left(x^{\prime}, y^{\prime}\right) / 2$ and $\left(\gamma_{\|}-\gamma_{\perp}\right) \Delta I\left(x^{\prime}, y^{\prime}\right) / 2$ are enough small, Equation 11 can be approximately rewritten as

$$
\begin{aligned}
H\left(x^{\prime},\right. & \left.y^{\prime}\right) U_{3, \mathrm{R}}^{\prime}\left(x^{\prime}, y^{\prime}\right) \\
& =\frac{U_{3, \mathrm{R}}^{\prime}\left(x^{\prime}, y^{\prime}\right)}{\sqrt{2}} \\
& \cdot\left\{\left[\begin{array}{c}
1 \\
-\mathrm{i}
\end{array}\right]\right. \\
& \left.+\mathrm{i}\left(\gamma_{\|}-\gamma_{\perp}\right) A_{3, \mathrm{~S}}\left(x^{\prime}, y^{\prime}\right) A_{3, \mathrm{R}}\left(x^{\prime}, y^{\prime}\right) \exp \left\{\mathrm{i}\left[\phi_{3, \mathrm{~S}}\left(x^{\prime}, y^{\prime}\right)-\phi_{3, \mathrm{R}}\left(x^{\prime}, y^{\prime}\right)\right]\right\}\left[\begin{array}{c}
1 \\
\mathrm{i}
\end{array}\right]\right\} \\
& =\frac{U_{3, \mathrm{R}}{ }^{\prime}\left(x^{\prime}, y^{\prime}\right)}{\sqrt{2}}\left\{\left[\begin{array}{c}
1 \\
-\mathrm{i}
\end{array}\right]+\mathrm{i}\left(\gamma_{\|}-\gamma_{\perp}\right) U_{3, \mathrm{~S}}\left(x^{\prime}, y^{\prime}\right) U_{3, \mathrm{R}}{ }^{*}\left(x^{\prime}, y^{\prime}\right)\left[\begin{array}{l}
1 \\
\mathrm{i}
\end{array}\right]\right\}
\end{aligned}
$$

The first and the second terms of Equation 14 are the zero-order beam with left-circular polarization and the first-order diffracted beam with right-circular polarization, respectively. The reconstructed signal is included in the first-order diffracted beam. The reconstructed signal beam is expressed by

$$
U_{3, \mathrm{~S}}^{\prime}\left(x^{\prime}, y^{\prime}\right)=\mathrm{i} \Gamma U_{3, \mathrm{~S}}\left(x^{\prime}, y^{\prime}\right) U_{3, \mathrm{R}}^{*}\left(x^{\prime}, y^{\prime}\right) U_{3, \mathrm{R}}^{\prime}\left(x^{\prime}, y^{\prime}\right)
$$


where $\Gamma$ is a coefficient dependent on the sensitivity of recording material and exposure time. At the Fourier plane, $U_{3, \mathrm{~S}}$ is Fourier transform pattern of $U_{\mathrm{S}}$ and represented by

$$
U_{3, \mathrm{~S}}\left(x^{\prime}, y^{\prime} ; z=0\right)=\iint U_{\mathrm{S}}(x, y) \exp \left[-\mathrm{i} 2 \pi\left(v_{x}{ }^{\prime} x+v_{y}{ }^{\prime} y\right)\right] \mathrm{d} x \mathrm{~d} y
$$

where

$$
\begin{aligned}
& v_{x}^{\prime}=\frac{x^{\prime}}{\lambda f} \\
& v_{y}^{\prime}=\frac{y^{\prime}}{\lambda f}
\end{aligned}
$$

Here, $\lambda$ and $f$ are wavelength of recording laser and focal length of FTL1, respectively. Equation 16 can be rewritten as

$$
U_{3, \mathrm{~S}}\left(x^{\prime}, y^{\prime} ; z=0\right)=\iint U_{\mathrm{S}}\left(-v_{x},-v_{y}\right) \exp \left[\mathrm{i} 2 \pi\left(v_{x} x^{\prime}+v_{y} y^{\prime}\right)\right] \mathrm{d} v_{x} \mathrm{~d} v_{y}
$$

where

$$
\begin{aligned}
& v_{x}=-\frac{x}{\lambda f} \\
& v_{y}=-\frac{y}{\lambda f}
\end{aligned}
$$

Equation 19 shows plane wave expansion of the complex amplitude of signal beam at Fourier plane. Therefore, $U_{3, \mathrm{~S}}$ at arbitrary position is expressed by

$$
=\iint U_{\mathrm{S}}\left(-v_{x},-v_{y}\right) \exp \left[\mathrm{i} 2 \pi\left(v_{x} x^{\prime}+v_{y} y^{\prime}+z \sqrt{\frac{1}{\lambda^{2}}-v_{x}^{2}-v_{y}^{2}}\right)\right] \mathrm{d} v_{x} \mathrm{~d} v_{y}
$$

In the case of $U_{\mathrm{R}}=A_{\mathrm{R}}, U_{3, \mathrm{~S}}$ is similarly expressed by

$$
U_{3, \mathrm{R}}\left(x^{\prime}, y^{\prime} ; z\right)=\iint A_{\mathrm{R}} \exp \left[\mathrm{i} 2 \pi\left(v_{x} x^{\prime}+v_{y} y^{\prime}+z \sqrt{\frac{1}{\lambda^{2}}-v_{x}^{2}-v_{y}^{2}}\right)\right] \mathrm{d} v_{x} \mathrm{~d} v_{y}
$$

In this study, it is considered that the position of an illumination beam is shifted by $\left(x_{\mathrm{R}}{ }^{\prime}, y_{\mathrm{R}}{ }^{\prime}\right)$. Then, $U_{3, \mathrm{R}^{\prime}}$ is expressed by

$$
\begin{aligned}
& U_{3, \mathrm{R}}{ }^{\prime}\left(x^{\prime}, y^{\prime} ; z\right) \\
& \quad=\iint A_{\mathrm{R}} \exp \left\{\mathrm{i} 2 \pi\left[v_{x}\left(x^{\prime}-x_{\mathrm{R}}{ }^{\prime}\right)+v_{y}\left(y^{\prime}-y_{\mathrm{R}}{ }^{\prime}\right)+z \sqrt{\frac{1}{\lambda^{2}}-v_{x}{ }^{2}-v_{y}{ }^{2}}\right]\right\} \mathrm{d} v_{x} \mathrm{~d} v_{y}
\end{aligned}
$$

In the case of $\left|v_{x}\right|,\left|v_{y}\right| \ll 1 / \lambda$, that is, $|x|,|y| \ll f, U_{3, \mathrm{~S}}, U_{3, \mathrm{R}}$, and $U_{3, \mathrm{R}}{ }^{\prime}$ are approximated as

$$
\begin{gathered}
U_{3, \mathrm{~S}}\left(x^{\prime}, y^{\prime} ; z\right) \\
=\iint U_{\mathrm{S}}\left(-v_{x},-v_{y}\right) \exp \left\{\mathrm{i} 2 \pi\left[v_{x} x^{\prime}+v_{y} y^{\prime}+\frac{z}{\lambda}-\frac{\lambda z}{2}\left(v_{x}^{2}+v_{y}{ }^{2}\right)\right]\right\} \mathrm{d} v_{x} \mathrm{~d} v_{y}
\end{gathered}
$$




$$
\begin{aligned}
& U_{3, \mathrm{R}}\left(x^{\prime}, y^{\prime} ; z\right)=\iint A_{\mathrm{R}} \exp \left\{\mathrm{i} 2 \pi\left[v_{x} x^{\prime}+v_{y} y^{\prime}+\frac{z}{\lambda}-\frac{\lambda z}{2}\left(v_{x}^{2}+v_{y}^{2}\right)\right]\right\} \mathrm{d} v_{x} \mathrm{~d} v_{y} \\
& =-\frac{\mathrm{i} A_{\mathrm{R}}}{\lambda z} \exp \left(\mathrm{i} \pi \frac{x^{\prime 2}+y^{\prime 2}}{\lambda z}+\frac{\mathrm{i} 2 \pi z}{\lambda}\right) \\
& =\iint A_{\mathrm{R}} \exp \left\{\mathrm{i} 2 \pi\left[v_{x}\left(x^{\prime}-x_{\mathrm{R}}^{\prime}\right)+v_{y}\left(y^{\prime}-y_{\mathrm{R}}^{\prime}\right)+\frac{z}{\lambda}-\frac{\lambda z}{2}\left(v_{x}^{2}+v_{y}^{2}\right)\right]\right\} \mathrm{d} v_{x} \mathrm{~d} v_{y} \\
& =-\frac{\mathrm{i} A_{\mathrm{R}}}{\lambda z} \exp \left[\mathrm{i} \pi \frac{\left(x^{\prime}-x_{\mathrm{R}}^{\prime}\right)^{2}+\left(y^{\prime}-y_{\mathrm{R}}^{\prime}\right)^{2}}{\lambda z}+\frac{\mathrm{i} 2 \pi z}{\lambda}\right]
\end{aligned}
$$

The complex amplitude of the reconstructed signal beam generated from a plane $z$ is expressed by

$$
\begin{aligned}
& U_{3, \mathrm{~S}}{ }^{\prime}\left(x^{\prime}, y^{\prime} ; z ; 0\right) \\
& =\mathrm{i} \Gamma\left(\frac{A_{\mathrm{R}}}{\lambda z}\right)^{2} \exp \left(\mathrm{i} \pi \frac{x_{\mathrm{R}}{ }^{2}+y_{\mathrm{R}}{ }^{2}-2 x_{\mathrm{R}}^{\prime} x^{\prime}-2 y_{\mathrm{R}}^{\prime} y^{\prime}}{\lambda z}\right) \iint U_{\mathrm{S}}\left(-v_{x},-v_{y}\right) \exp \left\{\mathrm { i } 2 \pi \left[v_{x} x^{\prime}\right.\right. \\
& \left.\left.+v_{x} y^{\prime}+\frac{z}{\lambda}-\frac{\lambda z}{2}\left(v_{x}^{2}+v_{y}^{2}\right)\right]\right\} \mathrm{d} v_{x} \mathrm{~d} v_{y} \\
& =\mathrm{i} \Gamma\left(\frac{A_{\mathrm{R}}}{\lambda z}\right)^{2} \exp \left(\mathrm{i} \pi \frac{x_{\mathrm{R}}{ }^{2}+y_{\mathrm{R}}^{\prime 2}-2 x_{\mathrm{R}}^{\prime} x^{\prime}-2 y_{\mathrm{R}}^{\prime} y^{\prime}}{\lambda z}\right) \mathrm{iFT}\left\{U _ { \mathrm { S } } ( - v _ { x } , - v _ { y } ) \operatorname { e x p } \left\{\mathrm { i } 2 \pi \left[\frac{z}{\lambda}\right.\right.\right. \\
& \left.\left.\left.-\frac{\lambda z}{2}\left(v_{x}^{2}+v_{y}^{2}\right)\right]\right\}\right\}\left(x^{\prime}, y^{\prime} ; z\right),
\end{aligned}
$$

where iFT denotes inverse Fourier transform. When the reconstructed signal beam is propagated by $z^{\prime}$, the complex amplitude is expressed by

$$
\begin{aligned}
& U_{3, \mathrm{~S}}{ }^{\prime}\left(x^{\prime}, y^{\prime} ; z ; z^{\prime}\right) \\
& =\iint \mathrm{FT}\left[U_{3, \mathrm{~S}^{\prime}}\left(x^{\prime}, y^{\prime} ; z ; 0\right)\right]\left(v_{x}, v_{y}\right) \exp \left\{\mathrm { i } 2 \pi \left[v_{x} x^{\prime}+v_{y} y^{\prime}\right.\right. \\
& \left.\left.+z^{\prime} \sqrt{\frac{1}{\lambda^{2}}-v_{x}^{2}-v_{y}^{2}}\right]\right\} \mathrm{d} v_{x} \mathrm{~d} v_{y} \\
& \approx \iint \mathrm{FT}\left[U_{3, \mathrm{~S}^{\prime}}\left(x^{\prime}, y^{\prime} ; z ; 0\right)\right]\left(v_{x}, v_{y}\right) \exp \left\{\mathrm { i } 2 \pi \left[v_{x} x^{\prime}+v_{y} y^{\prime}-\frac{z^{\prime}}{\lambda}\right.\right. \\
& \left.\left.+\frac{\lambda z^{\prime}}{2}\left(v_{x}^{2}+v_{y}^{2}\right)\right]\right\} \mathrm{d} v_{x} \mathrm{~d} v_{y} \\
& =\mathrm{i} \Gamma\left(\frac{A_{\mathrm{R}}}{\lambda z}\right)^{2} \exp \left(\mathrm{i} \pi \frac{x_{\mathrm{R}}{ }^{2}+y_{\mathrm{R}}{ }^{\prime 2}}{\lambda z}\right) \iint U_{\mathrm{S}}\left(-v_{x}-\frac{x_{\mathrm{R}}^{\prime}}{\lambda z},-v_{y}\right. \\
& \left.-\frac{y_{\mathrm{R}}^{\prime}}{\lambda z}\right) \exp \left\{\mathrm { i } 2 \pi \left\{v_{x} x^{\prime}+v_{y} y^{\prime}-\frac{z+z^{\prime}}{\lambda}\right.\right. \\
& \left.\left.+\frac{\lambda\left(z+z^{\prime}\right)}{2}\left[\left(v_{x}+\frac{x_{\mathrm{R}}^{\prime}}{\lambda z}\right)^{2}+\left(v_{y}+\frac{y_{\mathrm{R}}^{\prime}}{\lambda z}\right)^{2}\right]\right\}\right\} \mathrm{d} v_{x} \mathrm{~d} v_{y}
\end{aligned}
$$

where FT denotes Fourier transform. In the case of $z^{\prime}=-z$, Equation 29 is rewritten as 


$$
\begin{aligned}
& U_{3, \mathrm{~S}}^{\prime}\left(x^{\prime}, y^{\prime} ; z ;-z\right) \\
& =\mathrm{i} \Gamma\left(\frac{A_{\mathrm{R}}}{\lambda z}\right)^{2} \exp \left(\mathrm{i} \pi \frac{x_{\mathrm{R}}{ }^{2}+y_{\mathrm{R}}{ }^{2}}{\lambda z}\right) \iint U_{\mathrm{S}}\left(-v_{x}-\frac{x_{\mathrm{R}}^{\prime}}{\lambda z},-v_{y}\right. \\
& \left.-\frac{y_{\mathrm{R}}^{\prime}}{\lambda z}\right) \exp \left[\mathrm{i} 2 \pi\left(v_{x} x^{\prime}+v_{y} y^{\prime}\right)\right] \mathrm{d} v_{x} \mathrm{~d} v_{y} \\
& =\mathrm{i} \Gamma\left(\frac{A_{\mathrm{R}}}{\lambda z}\right)^{2} \exp \left(\mathrm{i} \pi \frac{x_{\mathrm{R}}{ }^{2}+y_{\mathrm{R}}{ }^{2}}{\lambda z}\right) \iint U_{\mathrm{S}}\left(x-\frac{f}{z} x_{\mathrm{R}}^{\prime}, y\right. \\
& \left.-\frac{f}{z} y_{\mathrm{R}}^{\prime}\right) \exp \left[-\mathrm{i} 2 \pi\left(v_{x}^{\prime} x+v_{y}^{\prime} y\right)\right] \mathrm{d} x \mathrm{~d} y \\
& =\mathrm{i} \Gamma\left(\frac{A_{\mathrm{R}}}{\lambda z}\right)^{2} \exp \left(\mathrm{i} \pi \frac{x_{\mathrm{R}}{ }^{2}+y_{\mathrm{R}}{ }^{2}}{\lambda z}\right) \mathrm{FT}\left[U_{\mathrm{S}}\left(x-\frac{f}{z} x_{\mathrm{R}}^{\prime}, y-\frac{f}{z} y_{\mathrm{R}}^{\prime}\right)\right]\left(v_{x}^{\prime}, v_{y}^{\prime}\right)
\end{aligned}
$$

By inverse Fourier transform of Equation 30 using FTL2, the complex amplitude of the reconstructed signal beam is written at image plane 2 as

$$
U_{4, \mathrm{~S}}^{\prime}(x, y ; z)=\mathrm{i} \Gamma\left(\frac{A_{\mathrm{R}}}{\lambda z}\right)^{2} \exp \left(\mathrm{i} \pi \frac{x_{\mathrm{R}}{ }^{2}+y_{\mathrm{R}}{ }^{2}}{\lambda z}\right) U_{\mathrm{S}}\left(x-\frac{f}{z} x_{\mathrm{R}}^{\prime}, y-\frac{f}{z} y_{\mathrm{R}}^{\prime}\right)
$$

As shown in Equation 31, the position of the reconstructed image and the phase are shifted corresponding to $\left(x_{\mathrm{R}}^{\prime}, y_{\mathrm{R}}^{\prime}\right), z$ and $f$. Equation 31 shows the case of the thickness of the recording medium is thin, that is, the recording medium is located at only $z$. Here, the surface position and the back position of the recording medium are respectively defined as $z_{1}$ and $z_{2}\left(z_{1}<z_{2}, z_{2}<0\right)$. Then, the reconstructed signal beam is expressed by

$$
U_{\mathrm{S}}^{\prime}(x, y)=\int_{z_{1}}^{z_{2}} \mathrm{i} \Gamma\left(\frac{A_{\mathrm{R}}}{\lambda z}\right)^{2} \exp \left(\mathrm{i} \pi \frac{x_{\mathrm{R}}^{\prime 2}+y_{\mathrm{R}}^{\prime 2}}{\lambda z}\right) U_{\mathrm{S}}\left(x-\frac{f}{z} x_{\mathrm{R}}^{\prime}, y-\frac{f}{z} y_{\mathrm{R}}^{\prime}\right) \mathrm{d} z
$$

When $x_{\mathrm{R}}{ }^{\prime}=0$ and $y_{\mathrm{R}}{ }^{\prime}=0$, the reconstructed signal beam is expressed by

$$
U_{\mathrm{S}}^{\prime}(x, y)=\int_{z_{1}}^{z_{2}} \mathrm{i} \Gamma\left(\frac{A_{\mathrm{R}}}{\lambda z}\right)^{2} U_{\mathrm{S}}(x, y) \mathrm{d} z=\frac{\mathrm{i} \Gamma A_{\mathrm{R}}^{2}\left(z_{2}-z_{1}\right) U_{\mathrm{S}}(x, y)}{\lambda^{2} z_{1} z_{2}}
$$

As shown in Equation 33, the recorded signal can be reconstructed. In retardagraphy, the phase pattern must be extracted. The phase pattern can be extracted by phase shifting algorithm using a transmitted illumination beam. The polarization state of the illumination beam is orthogonal to reconstructed signal beam. To use the phase shifting algorism, the polarization states of reconstructed signal beam and transmitted illumination beam are converted into two orthogonal linear polarizations and the phase difference is shifted by using a variable retarder. Then, two polarization components are interfered by using an analyzer and captured using an image sensor. The phase of the reconstructed signal beam is extracted from some captured images by a phase-shifting algorithm.

Next, the case of $x_{\mathrm{R}}{ }^{\prime} \neq 0$ and/or $y_{\mathrm{R}}{ }^{\prime} \neq 0$ is considered. When $U_{\mathrm{S}}$ is a plane wave as $A_{\mathrm{S}} \exp \left[\mathrm{i} 2 \pi\left(x_{\mathrm{S}}{ }^{\prime} x / \lambda f+y_{\mathrm{S}}{ }^{\prime} x / \lambda f\right)\right]$, Equation 32 is written as 


$$
\begin{aligned}
& U_{\mathrm{S}}^{\prime}(x, y) \\
& =\int_{z_{1}}^{z_{2}} \mathrm{i} \Gamma\left(\frac{A_{\mathrm{R}}}{\lambda z}\right)^{2} \exp \left(\mathrm{i} \pi \frac{x_{\mathrm{R}}{ }^{\prime 2}+y_{\mathrm{R}}{ }^{\prime 2}}{\lambda z}\right) \exp \left\{\mathrm { i } 2 \pi \left[\frac{x_{\mathrm{S}}{ }^{\prime}}{\lambda f}\left(x-\frac{f}{z} x_{\mathrm{R}}^{\prime}\right)\right.\right. \\
& \left.\left.+\frac{y_{\mathrm{S}}{ }^{\prime}}{\lambda f}\left(y-\frac{f}{z} y_{\mathrm{R}}^{\prime}\right)\right]\right\} \mathrm{d} z \\
& =-\frac{\Gamma A_{\mathrm{R}}^{2} A_{\mathrm{S}} \exp \left[\mathrm{i} 2 \pi\left(x_{\mathrm{S}}^{\prime} x / \lambda f+y_{\mathrm{S}}^{\prime} y / \lambda f\right)\right]}{\lambda \pi\left(x_{\mathrm{R}}{ }^{\prime 2}+y_{\mathrm{R}}{ }^{\prime 2}+2 x_{\mathrm{S}}^{\prime} x_{\mathrm{R}}{ }^{\prime}+2 y_{\mathrm{S}}^{\prime} y_{\mathrm{R}}^{\prime}\right)} \\
& \cdot\left[\exp \left(\mathrm{i} \pi \frac{x_{\mathrm{R}}^{\prime 2}+y_{\mathrm{R}}^{\prime 2}+2 x_{\mathrm{S}}^{\prime} x_{\mathrm{R}}^{\prime}+2 y_{\mathrm{S}}^{\prime} y_{\mathrm{R}}^{\prime}}{\lambda z_{2}}\right)\right. \\
& \left.-\exp \left(\mathrm{i} \pi \frac{x_{\mathrm{R}}{ }^{\prime 2}+y_{\mathrm{R}}{ }^{\prime 2}+2 x_{\mathrm{S}}^{\prime} x_{\mathrm{R}}{ }^{\prime}+2 y_{\mathrm{S}}{ }^{\prime} y_{\mathrm{R}}{ }^{\prime}}{\lambda z_{1}}\right)\right] \\
& =-\frac{\Gamma A_{\mathrm{R}}{ }^{2} A_{\mathrm{S}} \exp \left[\mathrm{i} 2 \pi\left(x_{\mathrm{S}}{ }^{\prime} x / \lambda f+y_{\mathrm{S}}{ }^{\prime} y / \lambda f\right)\right]}{\lambda \pi\left(x_{\mathrm{R}}{ }^{2}+y_{\mathrm{R}}{ }^{\prime 2}+2 x_{\mathrm{S}}^{\prime} x_{\mathrm{R}}{ }^{\prime}+2{y_{\mathrm{S}}}^{\prime} y_{\mathrm{R}}{ }^{\prime}\right)} \exp \left[\mathrm { i } \pi \frac { z _ { 1 } + z _ { 2 } } { 2 \lambda z _ { 1 } z _ { 2 } } \left(x_{\mathrm{R}}{ }^{2}+y_{\mathrm{R}}{ }^{\prime 2}\right.\right. \\
& \left.\left.+2 x_{\mathrm{S}}^{\prime} x_{\mathrm{R}}^{\prime}+2 y_{\mathrm{S}}^{\prime} y_{\mathrm{R}}{ }^{\prime}\right)\right] \\
& \cdot\left\{\exp \left[-\mathrm{i} \pi \frac{z_{2}-z_{1}}{2 \lambda z_{1} z_{2}}\left(x_{\mathrm{R}}{ }^{2}+y_{\mathrm{R}}{ }^{\prime 2}+2 x_{\mathrm{S}}^{\prime} x_{\mathrm{R}}{ }^{\prime}+2 y_{\mathrm{S}}{ }^{\prime} y_{\mathrm{R}}{ }^{\prime}\right)\right]\right. \\
& \left.-\exp \left[\mathrm{i} \pi \frac{z_{2}-z_{1}}{2 \lambda z_{1} z_{2}}\left(x_{\mathrm{R}}{ }^{2}+y_{\mathrm{R}}{ }^{\prime 2}+2 x_{\mathrm{S}}^{\prime} x_{\mathrm{R}}{ }^{\prime}+2 y_{\mathrm{S}}{ }^{\prime} y_{\mathrm{R}}{ }^{\prime}\right)\right]\right\}
\end{aligned}
$$

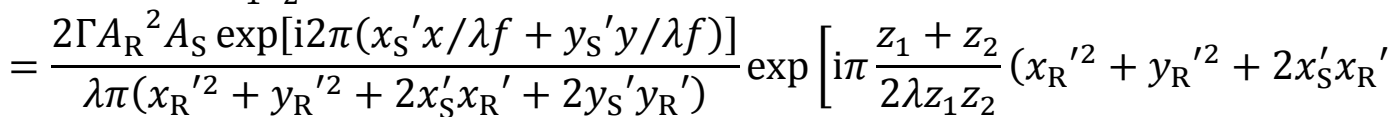

$$
\begin{aligned}
& \left.\left.+2 y_{\mathrm{S}}^{\prime} y_{\mathrm{R}}^{\prime}\right)\right] \sin \left[\pi \frac{z_{2}-z_{1}}{2 \lambda z_{1} z_{2}}\left(x_{\mathrm{R}}{ }^{2}+y_{\mathrm{R}}^{\prime 2}+2 x_{\mathrm{S}}^{\prime} x_{\mathrm{R}}^{\prime}+2 y_{\mathrm{S}}^{\prime} y_{\mathrm{R}}^{\prime}\right)\right]
\end{aligned}
$$

Therefore, $U_{\mathrm{S}}^{\prime}$ becomes zero in case of

$$
\frac{z_{2}-z_{1}}{2 \lambda z_{1} z_{2}}\left(x_{\mathrm{R}}{ }^{2}+y_{\mathrm{R}}{ }^{2}+2 x_{\mathrm{S}}^{\prime} x_{\mathrm{R}}^{\prime}+2 y_{\mathrm{S}}^{\prime} y_{\mathrm{R}}{ }^{\prime}\right)=m
$$

where $m$ is an integer except for zero. In the case of $m=1$ and $y_{\mathrm{R}}^{\prime}=0, x_{\mathrm{R}}^{\prime}$ is expressed by

$$
x_{\mathrm{R}}^{\prime}=-x_{\mathrm{S}}^{\prime} \pm \sqrt{x_{\mathrm{S}}^{\prime 2}+\frac{2 \lambda z_{1} z_{2}}{z_{2}-z_{1}}}
$$

From Equation 36, it is found that shift multiplex recording is realized in volume retardagraphy.

\section{Experimental Procedure}

Figure 2 shows the experimental setup for volume retardagraphy. A diode laser with a wavelength of $406 \mathrm{~nm}$ was uses for recording and reconstruction. A liquid crystal on silicon spatial light modulator (SLM; Hamamatsu X10408-5) was used to give data pages to recording beam. The polarization state of the laser beam after QWP1 is adjusted to linear with an azimuthal angle by rotating a half-wave plate (HWP1) and QWP1. By using QWP1, $p$ - and $s$-polarization components were converted into right- and left-circular polarization components, respectively. In this setup, when the homogeneous image was inputted to the SLM and the pixel value was changed, the azimuthal angle of the linear polarization was changed corresponding to the pixel value because the phase difference between rightand left-circular polarization components were changed. The size of patterns modulated by the SLM 
was reduced by two lenses and the beam was focused by using an objective lens. A polarization-sensitive recording medium made of 9,10-phentanthrenenequinone doped polymethylmethacrylate (PQ-PMMA) was put between two objective lenses. The thickness of the medium was $1 \mathrm{~mm}$. Then, the position of the back of the medium was set to the position of the Fourier plane of the recording pattern. The recording beam was illuminated onto the medium. The power of the recording beam at the recording plane and the irradiation time were set to $8 \mathrm{~mW}$ and $33 \mathrm{~ms}$, respectively.

Figure 2. Experimental setup for volume retardagraphy. L, OL, M, HWP, QWP, P, A, SLM, VR are lens, objective lens, mirror, half-wave plate, quarter-wave plate, polarizer, analyzer, spatial light modulator, variable retarder, respectively. Focal lengths $(\mathrm{mm})$ are denoted around lenses. The polarizer is used in only reconstruction.

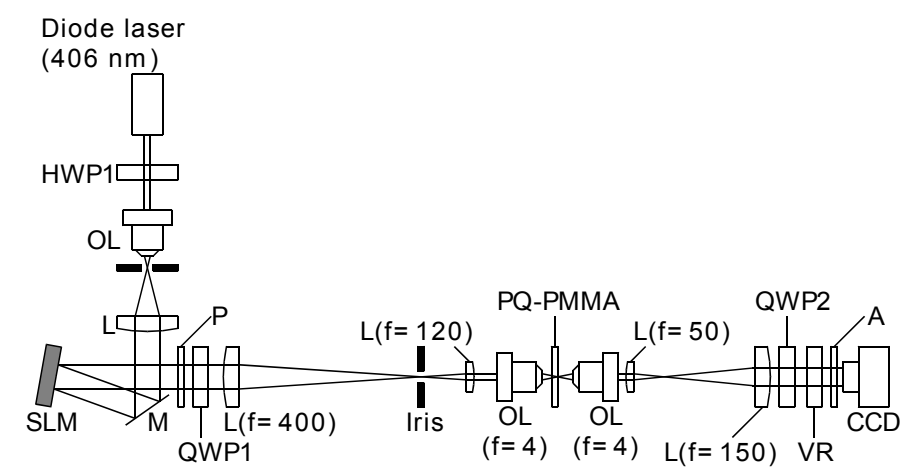

In recording, a phase data page as shown in Figure 3 was displayed on the SLM. In the SLM, the phase of $p$-polarization component was modulated corresponding to the phase data page. The phase data page consisted of $15 \times 14$ symbols. A symbol consisted of $2 \times 2$ data pixels. The data pixel consisted of $10 \times 10$ pixels of SLM. The pixel size of SLM is $20 \mu \mathrm{m} \times 20 \mu \mathrm{m}$. The phase value was four-valued and the valued of $0,0.5 \pi, 1.0 \pi, 1.5 \pi$ rad was used. In a symbol, the phase value was set as the four values were different each other. Then, a symbol can express 24 cases, that is, the information amount of symbol is more than 4 bits. Therefore, a recording digital data was divided by every 4 bits and converted into phase data pages. In this experiment, 12 phase data pages were recorded in multiple by laterally shifting the position of the medium by using a linear stage (SIGMA KOKI SGSP20-20(X)). The shift amount was set to $100 \mu \mathrm{m}$.

In reconstruction, a homogeneous pattern was displayed on SLM and a polarizer was put between the SLM and the QWP1. The transmission axis of the polarizer was set as s-polarization component is transmitted. Then, the $s$-polarized beam was converted into left circular polarized beam by using QWP1. The recorded phase data pages were reconstructed by illuminating the left circular polarized beam. Then, a reconstructed signal beam with right-circular polarization was generated from the recorded medium. The reconstructed signal beam was imaged on a CCD plane by using an objective lens and telecentric optical system. The right- and left-circular polarization components were converted into $45^{\circ}$ and $-45^{\circ}$ linear polarization components by using QWP2. The reconstructed signal beam with $45^{\circ}$ linear polarization and the transmitted illumination beam with $-45^{\circ}$ linear polarization were interfered through an analyzer. Therefore, an interference pattern between reconstructed signal beam and transmitted illumination beam was captured using the CCD camera. Then, the phase between the 
reconstructed signal beam and the transmitted illumination beam was shifted by using a liquid crystal variable retarder (Meadowlark LDC-100) and four images were captured. By using the four step phase-shifting algorithm, the complex amplitude of reconstructed signal beam was extracted. Furthermore, pixels between reconstructed data page and CCD camera were matched by cubic spline interpolation. Then, the phase was calculated from the complex amplitude.

Figure 3. Phase data page. (a) four-valued phase data phase; (b) four symbols.

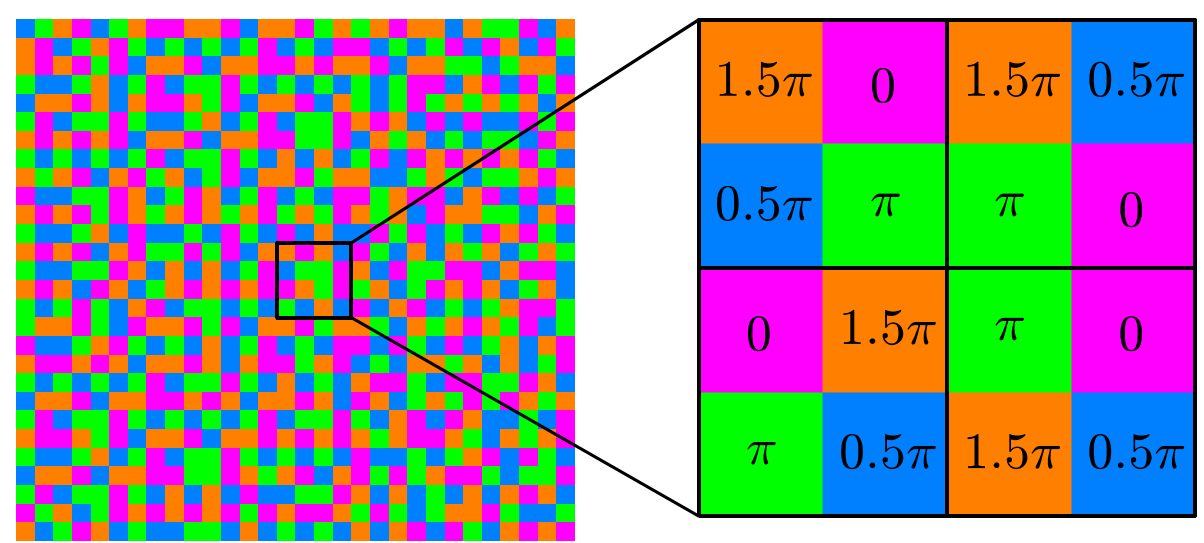

(a)

(b)

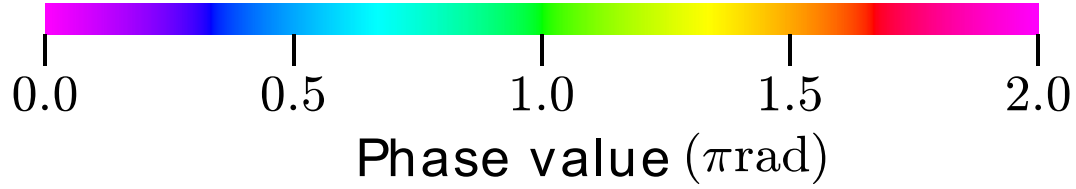

\section{Results and Discussion}

Figure $4 \mathrm{~b}$ shows a reconstructed phase pattern. The phase value is shifted by $\pi / 4$. The shift value is required by correcting initial phase in recording. It does not seem that the reconstructed phase pattern is similar to recording data page (Figure 4a). The extinction ratio of right-circular polarization component to left-circular polarization component is not zero in this system. Therefore, the right-circular polarization component is slightly included in the illumination beam. The reconstructed signal beam is superposed with the right-circular polarization component of transmitted illumination beam. Therefore, the reconstructed phase value was changed. By considering the coding method, the transmitted illumination component can be eliminated. In the coding method, the average of complex amplitude of a symbol is always zero when it is assumed that the intensity is homogeneous. When the complex amplitude values of the reconstructed signal pattern in a symbol were averaged, the transmitted signal beam component is obtained. A data pixel consisted of $10 \times 10$ pixels. The central $6 \times 6$ pixels in the $10 \times 10$ pixels were used to average the complex amplitude. Then, the standard deviation of real and imaginary parts was calculated and an argument was calculated from the real and imaginary parts. When the phase difference at a pixel is larger than the argument value, the complex amplitude value at the pixel is not used to calculate average complex amplitude. By substituting the average complex amplitude from the reconstructed signal complex amplitude, corrected phase pattern is obtained. The corrected phase pattern is shown in Figure 4c. Furthermore, to evaluate symbol error rate (SER), the reconstructed phase pattern must be four-valued. The sine and cosine of the phase value are calculated 
in a symbol and compared with 16 cases of symbol pattern. Then, the phase pattern is substituted by a symbol pattern with the least square of the sum of the difference. By the method, a phase data page was reconstructed. The reconstructed phase data page is shown in Figure 4d. The symbol error rates (SERs) of reconstructed 12 phase data pages are shown Table 1. As shown in Table 1, SERs were under $11 \%$. Those values were enough low to make zero by using an error correction method. The symbol error rate is dependent on the resolution and the positioning accuracy of the linear stage because of shift selectivity. In this experiment, the resolution and the accuracy are $2 \mu \mathrm{m}$ and $10 \mu \mathrm{m}$, respectively.

Figure 4. Reconstructing process. (a) Recording phase data phase; (b) Reconstructed phase pattern; (c) Corrected phase pattern; (d) Reconstructed phase data page.

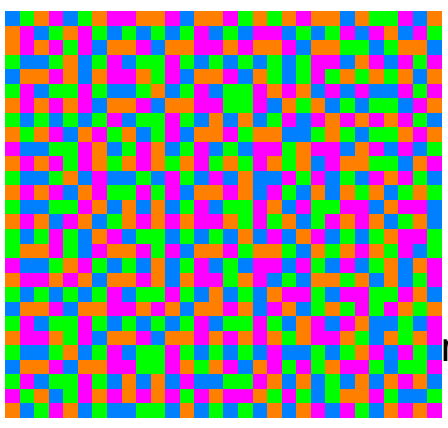

(a)
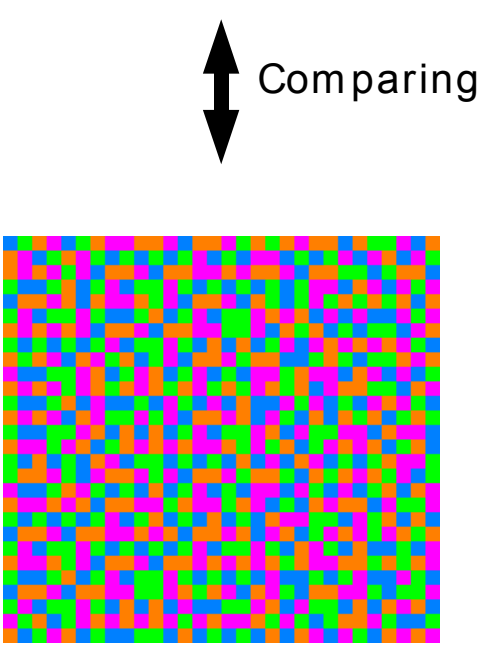

(d)

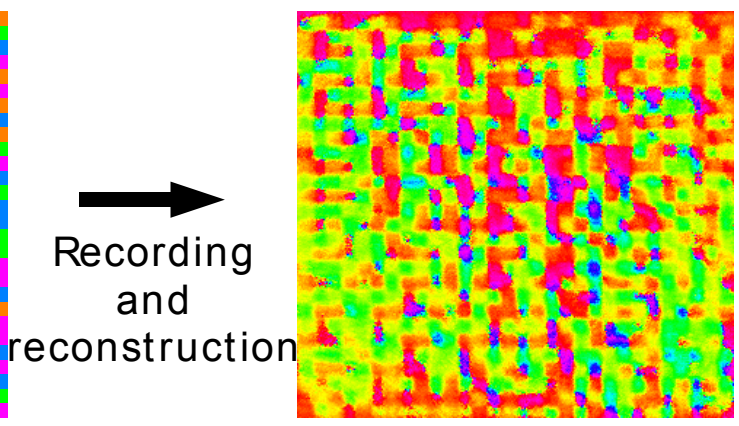

(b)

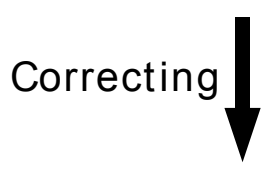

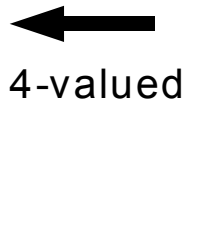

(c)

Table 1. Symbol error rate (SER) of reconstructed phase data pages.

\begin{tabular}{ccccccc}
\hline Page no. & $\mathbf{1}$ & $\mathbf{2}$ & $\mathbf{3}$ & $\mathbf{4}$ & $\mathbf{5}$ & $\mathbf{6}$ \\
\hline SER (\%) & 3.33 & 0 & 2.38 & 0.48 & 0 & 5.71 \\
Page no. & 7 & 8 & 9 & 10 & 11 & 12 \\
SER (\%) & 10.95 & 10.95 & 2.86 & 1.90 & 2.86 & 0.48 \\
\hline
\end{tabular}

In this study, the data pixel size of phase data pages was reduced from $200 \mu \mathrm{m}$ to $60 \mu \mathrm{m}$. Then, the width of the data page was calculated to $W=30 \times 60=1.8 \mathrm{~mm}$. When the recording medium is thin, that is, $z_{1}=z_{2}$, and it is located at $z_{1}=z_{2}=-1 \mathrm{~mm},\left|x_{\mathrm{R}}^{\prime}\right|=W\left|z_{1}\right| / f=450 \mu \mathrm{m}$ is required to record another phase data page. When the thickness becomes greater, that is $z_{2}$ approachess zero, the reconstructed image is eliminated by shifting the recording medium with less than $450 \mu \mathrm{m}$. Therefore, 
shift multiplex recording was realized with the shift amount of $100 \mu \mathrm{m}$. When the shift amount is decreased, the crosstalk noise is increased. Therefore, SER is increased. For a shift amount of $100 \mu \mathrm{m}$, it has been determined that the SERs fall below about 10\%. To achieve higher shift selectivity, the low spatial frequency components of recording phase data page must be removed. It is possible to decrease the value to remove the low spatial frequency components of signal beam pattern by using other coding methods of the phase data pages. In this study, a PQ-PMMA plate with a thickness of $1 \mathrm{~mm}$, which is easy to prepare, was used as a recording medium to experimentally verify the principle of volume retardagraphy. By improving the polarization-sensitivity of recording medium as well as shift selectivity, it is expected to increase data capacity and data transfer rate.

\section{Conclusions}

In this paper, some phase data pages were recorded and reconstructed by volume retardagraphy with shift multiplexing. The shift amount was set to $100 \mu \mathrm{m}$. From the result, it was verified that volume retardagraphy has shift selectivity. However, the shift selectivity of $100 \mu \mathrm{m}$ is low to apply a next-generation optical memory. By using a coding method without low spatial frequency components, the shift selectivity can be higher. Therefore, volume retardagraphy is expected to apply a next-generation optical memory with parallel recording and a simple optical system.

\section{Acknowledgments}

This work has been partially supported by the Japan Science and Technology Agency (JST) under the Strategic Promotion of Innovation Research and Development Program.

\section{Conflicts of Interest}

The authors declare no conflict of interest.

\section{References}

1. Curtis, K.; Dhar, L.; Hill, A.; Wilson, W.; Ayres, M. Holographic Data Storage: From Theory to Practical Systems; John Wiley \& Sons: Chichester, UK, 2010.

2. Horimai, H.; Tan, X.D.; Li, J. Collinear holography. Appl. Opt. 2005, 44, 2575-2579.

3. Shimura, T.; Ichimura, S.; Fujimura, R.; Kuroda, K.; Tan, X.D.; Horimai, H. Analysis of a collinear holographic storage system: Introduction of pixel spread function. Opt. Lett. 2006, 31, 1208-1230.

4. Fukuda, T.; Kim, J.Y.; Barada, D.; Yase, K. Photoinduced cooperative molecular reorientation on azobenzene side-chain-type copolymers. J. Photochem. Photobiol. A 2006, 183, 273-279.

5. Kawatsuki, N.; Takatsuka, H.; Yamamoto, T.; Sangen, O. Optical anisotropy of a photoreacted side-chain liquid-crystalline polymer induced by linearly polarized UV light. J. Polym. Sci. A 1997, 36, 1521-1526.

6. Trofimova, A.V.; Stankevich, A.I.; Mogilnyi, V.V. Phenanthrenequinone-polymethylmethacrylate composite for polarization phase recording. J. Appl. Spectrosc. 2009, 76, 585-591. 
7. Barada, D.; Tamura, K.; Fukuda, T.; Itoh, M.; Yatagai, T. Retardagraphy: A technique for optical recording of the retardance pattern of an optical anisotropic object on a polarization-sensitive film using a single beam. Opt. Lett. 2008, 33, 3007-3009.

8. Barada, D.; Tamura, K.; Fukuda, T.; Yatagai, T. Optical information recording in films of photoinduced birefringent materials and application to retardagraphy. Jpn. J. Appl. Phys. 2009, 48, 09LE02.

9. Barada, D.; Kawagoe, Y.; Tamura, K.; Fukuda, T.; Yatagai, T. Self-imaging properties of fresnel retardagram recorded on azobenzene film. Jpn. J. Appl. Phys. 2010, 49, 01 AD02.

10. Nikolova, L.; Ramanujam, P.S. Polarization Holography; Cambridge University Press: Cambridge, UK, 2009.

11. Barada, D.; Ochiai, T.; Fukuda, T.; Kawata, S.; Kuroda, K.; Yatagai, T. Dual-channel polarization holography: A technique for recording two complex amplitude components of a vector wave. Opt. Lett. 2012, 37, 4528-4530.

12. Ochiai, T.; Barada, D.; Fukuda, T.; Hayasaki, H.; Kuroda, K.; Yatagai, T. Angular multiplex recording of data pages by dual-channel polarization holography. Opt. Lett. 2013, 38, 748-750.

(C) 2014 by the authors; licensee MDPI, Basel, Switzerland. This article is an open access article distributed under the terms and conditions of the Creative Commons Attribution license (http://creativecommons.org/licenses/by/3.0/). 